\title{
Bio chitosan/nanodiamond gic from design to applications: in vitro investigation
}

\begin{abstract}
Background: Restoring the carious lesion at an early stage is an ideal treatment option in order to preserve the primary teeth until its normal anticipated exfoliation. This helps to assist in the maintenance of a healthy oral environment and arch length as well as to preserve the function of mastication and speech. However, even a simple restorative treatment plan is likely to evoke anxiety in a pediatric patient and may prove to be a challenge to the clinician. Hence when choice of the restorative material is made, simplicity of clinical application of the material should be considered along with other properties of the restorative material. The interest in the clinical use of glass ionomer cements (GIC) arose mainly from their particular advantage of requirement of a short time to fill the cavity which is a desirable property while treating young children.
\end{abstract}

Results: In general there was an increase in bond strength of the dentine treated with the modified GIC materials compared to the bond strength of the conventionally bonded teeth. Functionalized bio-materials showed the highest adhesive force, and this result can be expected because of the well-known intrinsic bio-adhesive properties of chitosan [Vasir 2002] All the test samples gave an average inhibition zone larger than the tetracycline control disc, thereby confirming the antibacterial activity of the different bio-active GIC's containing combinations against Stapylococcus aureus. Using the Student's T-test $(\mathrm{p}<0.01)$, there was a significant difference between the rest of the samples when compared to each other and the positive control. Based on the scores that were tabulated, the p-value was $>0.05$, thus indicative that there was no significant difference between micro-leakage of glass ionomer cement, chitosan modified glass ionomer cement, nanodiamond modified glass ionomer cement as well as chitosan: nanodiamond modified glass ionomer cements.

Conclusion: Incorporation of chitosan or nanodiamond or combination of Chitosan/ nanodiamond into the conventional GIC provides a promising multi-dimensional functional biomaterial with excellent properties such as increased shear bond strength, certain degree of microbiological properties, good bio-adhesion without compromising important properties such as microleakage.

Clinical significance: The interest in the clinical use of glass ionomer cements (GIC) arose mainly from their particular advantage of requirement of a short time to fill the cavity which is a desirable property while treating young children. The additional benefits of the application of bioactive GIC sealant materials lies in the build-in functionality of these materials to chemical attack in oral cavity as well as additional antibacterial action.
Volume 2 Issue 2 - 2017

Tamara PerchyonokV

Research and development, Australia

Correspondence: Tamara PerchyonokV, Research and development,VTPCHEM PTY LTD, Glenhuntly, Melbourne, 3163, Australia, Email tamaraperchyonok@gmail.com

Received: February 01, 2017| Published: March 31, 2017

\section{Introduction}

Dental caries is an age old disease and due to lack of oral health awareness and frequent ingestion of refined carbohydrates, caries is most commonly seen affecting pediatric patients. Restoring the carious lesion at an early stage is an ideal treatment option in order to preserve the primary teeth until its normal anticipated exfoliation. This helps to assist in the maintenance of a healthy oral environment and arch length as well as to preserve the function of mastication and speech. ${ }^{1}$ However, even a simple restorative treatment plan is likely to evoke anxiety in a pediatric patient and may prove to be a challenge to the clinician. Hence when choice of the restorative material is made, simplicity of clinical application of the material should be considered along with other properties of the restorative material. The interest in the clinical use of glass ionomer cements (GIC) arose mainly from their particular advantage of requirement of a short time to fill the cavity which is a desirable property while treating young children. ${ }^{2}$
Condensable or high-viscosity glass ionomer cements, developed early in the 1990s, as filling materials in the atraumatic restorative therapy technique, were desirable due to their advantageous properties like faster setting, adequate strength and polishability in a single visit. However, the risk of fracture exists for large restorations. ${ }^{3,4}$ Highviscosity glass ionomers are still inferior to resin-based restorative materials when it comes to fracture toughness. ${ }^{5}$ Hence, there has been a constant quest for further improvement in the properties of the material while retaining its multitude of clinical advantages.

It was reported in 2007 that the flexural strength of a commercial GIC was significantly improved by the addition of chitosan. Moreover, in the presence of chitosan, it was found that release of fluoride ions from GIC was catalyzed. ${ }^{6}$ Chitosan is a partially or completely deacetylated derivative of chitin. Researchers have demonstrated its great potential for a wide range of uses due to its versatile chemical and physical properties like biodegradability, biocompatibility, antimicrobial activity, non toxicity. ${ }^{7,8}$ 
However, addition of any agent into a material in an attempt to improve the properties, should not jeopardize any other desirable property of the parent material. An important property responsible for the success of a material used for restorative purposes in the oral cavity is its ability to bond to tooth structure in a way that there is a complete and perfect seal between the margins of restorations and tissue of the tooth. A measure of this property is microleakage. ${ }^{9}$ Glass ionomer cement has chemical bonding to tooth structure. Hence a good adhesion to tooth lowers the risk of microleakage at the margins. The addition of chitosan in an attempt to improve the physical properties should not interfere with the adhesive property and escalate the risk of microleakage at the margins of the restoration.

As our continuous interest in combining favorable bio-active properties of biological scaffolds such as chitosan and/or nanodiamonds with conventional dental materials, in order to enhance the properties, functionality and biological compatibility we aimed to report characterization and application of the newly developed chitosan and/or nano-diamond modified GIC materials. The detailed investigation into physical and chemical properties of these materials including the assessment of microleakage is reported here.

\section{Materials and methods}

Chitosan (Aldrich, Australia), glycerol (Sigma, USA), glacial acetic acid (E. Merck, Germany) were used as received. The degree of de-acetylation of typical commercial chitosan used in this study is $87 \%$. Chitosan with molecular weight $2.5 \times 103 \mathrm{KD}$ was used in the study. The isoelectric point is 4.0-5.0.

\section{Shear bond strength etch and no etch prototype}

Extracted human molars were used within 2 months of storage in water containing thymol crystals. Only undamaged teeth were selected. The roots of the teeth were removed and all the occlusal enamel exposed. The teeth were embedded in $10 \mathrm{~mm}$ length PVC (Consjit Tubing, SA PVC, JHB, RSA) pipes with cold cure acrylic resin so that the exposed enamel is projected well above the acrylic and the dentin then thoroughly washed under tap water. Conventional GIC (Fuji IX) and corresponding bio-active modified materials composite studs each with an internal diameter of $2.6 \mathrm{~mm}$ and height of $1 \mathrm{~mm}$, were bonded to the dentin surface of each tooth via application of dentin conditioner prior to applying and allowed to set following manufacturers specification for Fuji IX.

In this way, 40 tooth samples (each containing single studs) were prepared and divided at random into 5 groups of 8 each. The teeth were stored in a solution of artificial saliva. After $24 \mathrm{~h}$, the shear bond strength of one stud of each tooth was tested for failure (Zwick Universal Testing Machine, Germany) by means of a knife-edged rod at a crosshead speed of $0.5 \mathrm{~mm} / \mathrm{min}$. The other stud was tested after 6 months. All data were analyzed using the non-parametric ANOVA test.

\section{Microbiology investigations}

A type strain of Staphylococcus aureus (ATCC 12600), obtained from the American Type Culture Collection (Manassas, USA) was used as test bacterium for estimating the antibacterial activity of the hydrogels. The antibacterial activity of the prepared chitosan hydrogels were tested using the standard Kirby-Bauer agar disc diffusion method. 31,32 Five to $6 \mathrm{~mm}$ deep Muller-Hinton agar (Oxoid, Basingstoke, UK) plates were inoculated by streaking a standardized inoculum suspension that match a $0.5 \mathrm{McF}$ arland standard and containing $10^{7}-$ $10^{8}$ colony forming units $/ \mathrm{ml}$ with a throat cotton swab. For each test sample $500 \mu \mathrm{g}$ of hydrogel was applied to a $6 \mathrm{~mm}$ diameter paper disc. The paper discs were placed on the inoculated Muller-Hinton agar medium and incubated at $37^{\circ} \mathrm{C}$ for 24 hours. The diameter of the zones of growth inhibition was measured with a caliper. Each measurement was done in triplicate and the testing of each sample was repeated 3 times. The antibacterial efficacy of the prepared gels were compared to antibiotic sensitivity discs (Mast Laboratories, Merseyside UL) containing $30 \mu \mathrm{g}$ of Nystatin per disc.

\section{Bio adhesive study}

Bio-adhesion studies were done using Chatillon apparatus for force measurement. ${ }^{10}$ This method determines the maximum force and work needed to separate two surfaces in intimate contact. ${ }^{10}$ The hydrogels $(0.1 \mathrm{~g})$ were homogeneously spread on a $\mathrm{cm} 2$ glass disk and then the disks were fixed to the support of the tensile strength tester using double side adhesive. The gel was brought into contact with the contact with slice of enamel was established in order to imitate adhesion of the gel to the tooth structure, after a preset contact time ( $1 \mathrm{~min})$ under contact strength $(0.5 \mathrm{~N})$ the 2 surfaces were separated at a constant rate of displacement $(1 \mathrm{~mm} / \mathrm{s})$. The strength was recorded as a function of the displacement, which allowed to determine the maximal detachment force, Fmax, and the work of adhesion, W, which was calculated from the area under the strength-displacement curve.

\section{Collection of samples for microleakage investigations}

For the assessment of microleakage a total of one hundred and 20 sound third molar teeth were obtained. The extracted teeth were cleaned of soft tissue and debris and stored in artificial saliva at room temperature. ${ }^{9}$ The teeth were disinfected by immersion in $1 \%$ Thymol solution for 1 week and then washed and dried. ${ }^{11}$

The samples were divided into the following groups:

Group I: Glass ionomer cement (Fuji IX).

Group II: Chitosan modified glass ionomer cement.

Group III: Nanodiamond modified glass ionomer cement

Group IV: Chitosan: Nanodiamond modified glass ionomer cement

\section{Preparation of class $\mathbf{V}$ cavities}

Class V cavities $4 \mathrm{~mm}$ wide $\times 2 \mathrm{~mm}$ high $\times 1.5 \mathrm{~mm}$ deep 10 were prepared on the buccal surfaces of teeth with no retentive features incorporated in the cavity design, using burs(No. 1 round bur, No. 57 straight fissure bur) with high speed air rotor hand piece with water coolant. All cavosurface margins were kept at $90^{\circ} \mathrm{C}$ without bevel designs and burs were changed after every five preparations. ${ }^{11,12}$ The standardization of cavities was done using a divider, dial callipers, and a graduated probe to further confirm the depth of cavity.

\section{Restorations of the cavities in groups I, II, III and IV}

The prepared cavities were restored in all four groups with the respective materials after conditioning. GC dentin conditioner was applied for 20seconds to the cavity walls using a brush with light scrubbing motion, followed by rinsing and drying by directing the air stream from the sides to avoid the desiccation of dentin. The cavities were restored and varnish was applied. 


\section{Preparation for assessment of microleakage}

After restoration, the teeth was stored in artificial saliva at $37^{\circ} \mathrm{C}$ for 24 hours $^{13,14}$ and then subjected to 1500 thermo cycles 11 to 14 at $5^{\circ} \mathrm{C}$ and $60^{\circ} \mathrm{C}$, with 20 seconds of dwell time in each bath. ${ }^{12}$ Following thermo cycling the specimens were prepared for immersion in dye solution. All the tooth surfaces except the restoration and a $1 \mathrm{~mm}$ zone adjacent to its margins were covered with two coats of varnish. The root apices if any, were sealed with sticky wax. ${ }^{14}$ The coated teeth were then immersed in $2 \%$ basic fuchsin dye solution (Aldrich, Australia) for a period of 24 hours at $37^{\circ} \mathrm{C}$. After removal from the dye, the coatings were stripped from the teeth by peeling and where necessary, by scraping. The teeth were then thoroughly washed in water, dried and then were mounted in acrylic resin prior to sectioning. The teeth were sectioned into two halves bucco-lingually in an occluso-apical direction through the middle of restoration by using a diamond disk mounted on a straight hand piece with water coolant. Each section was then observed under a stereomicroscope (Leica M 80) with a magnification of $30 \times$. The degree of microleakage of both halves was assessed. The section showing the maximum degree of dye penetration was chosen for grading the microleakage.

The extent of the microleakage was noted according to the following scoring criteria 9:
1. No marginal leakage
2. Up to $1 / 3$ cavity depth
3. $1 / 3-2 / 3$ cavity depth
4. $>2 / 3$ cavity depth but not involving the axial wall.
5. Involving the axial wall.

\section{Results and discussion}

Figure 1A \& Figure 1B gives the shear bond strength values $(\mathrm{MPa})$ after 24hours of storage of samples in artificial saliva, respectively, using conventional GIC protocol discussed in the experimental section. In general there was an increase in bond strength of the dentine treated with the modified GIC materials compared to the bond strength of the conventionally bonded teeth.

An increase in the shear bond strength was also previously reported [Perchyonok, 2015] for chitosan containing bio-active materials. The additional advantage of the system may suggest that, chitosan: bioactive and nano-diamond interaction with crystalline hydroxyapatite structure of the dentine layer increases the dentin bond strength observed especially in the case of the direct bonding between the hydrogel and the enamel interface. ${ }^{13}$

\section{Bio adhesion}

Higher adhesiveness of the gels is desired to maintain an intimate contact with dentin structure and results are summarized in Table 1. Functionalized bio-materials showed the highest adhesive force, and this result can be expected because of the well-known intrinsic bioadhesive properties of chitosan. ${ }^{14}$

Chitosan hydrogels showed the highest adhesive force and the work of adhesion this can be expected because of the well-known intrinsic bio-adhesive properties of chitosan. The detailed investigation of the newly produced bioactive modified GIC's with particular attention being paid towards understanding of the exact nature of interaction between bio-actives such as nano-diamond, chitosan and dentin structure are currently on the way in our laboratory.

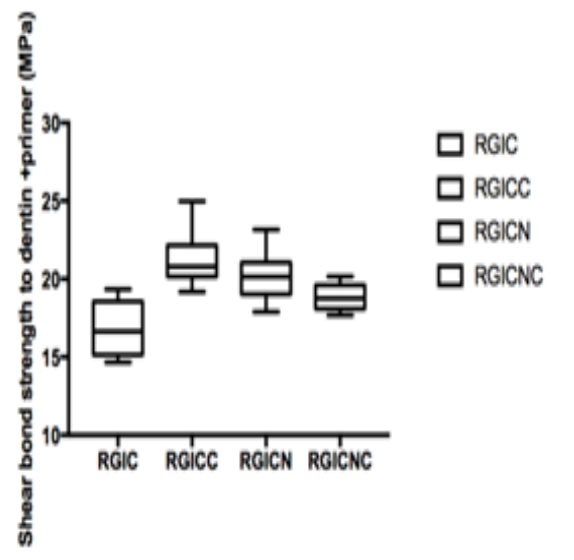

A. Dentin bonding with dentin conditioner after 24 hours de-bonding using conventional and modified GIC.

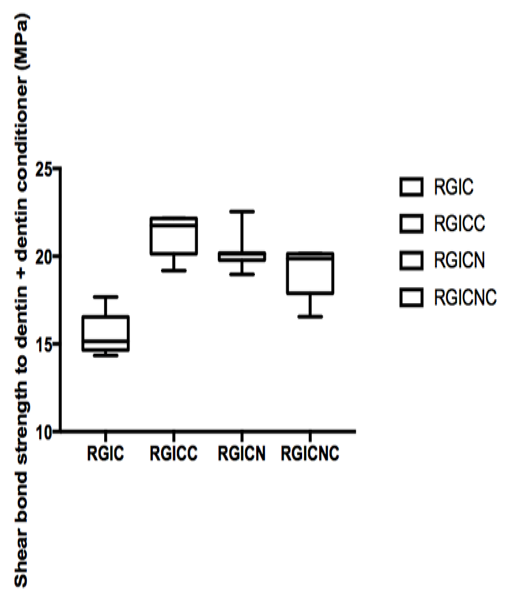

B. Dentin bonding with dentin conditioner after 6 month storage in artificial saliva and de-bonding.

Figure I Shear bond strength in $\mathrm{mPa}$

\section{Microbiology of the modified GIC}

All the test samples gave an average inhibition zone larger than the tetracycline control disc, thereby confirming the antibacterial activity of the different bio-active GIC's containing combinations against Stapylococcus aureus (Figure 2). ${ }^{15}$ Using the Student's T-test $(\mathrm{p}<0.01)$, there was a significant difference between the rest of the samples when compared to each other and the positive control.

The presence of $S$. aureus in the dental caries development and progression and therefore development the bio-active chitosan and nano-diamond modified GIC materials can contribute to development of protective fissure sealant materials and work is currently on the way in our laboratory to develop this materials further.

\section{Micro-leakage and modified GIC's detailed investiga- tion}

A majority of the samples in groups I, II, III and IV did not reveal 
any micro-leakage with a score of 0 as depicted in Figure 3 . Based on the scores that were tabulated, the p-value was $>0.05$, thus indicative that there was no significant difference between micro-leakage of glass ionomer cement, chitosan modified glass ionomer cement, nanodiamond modified glass ionomer cement as well as chitosan: nanodiamond modified glass ionomer cements.

Table I Adhesive strength in dentin

\begin{tabular}{lll}
\hline Modified RGIC compound & Adhesive force(N) $\mathbf{S D}($ dentin) & Work of adhesion(N.cm) $\pm \mathbf{S D}($ dentin) \\
\hline GICNC & $1.72 \pm 0.19$ & $7.4 \pm 0.15$ \\
GICN & $1.68 \pm 0.12$ & $7.24 \pm 0.23$ \\
GICC & $1.45 \pm 0.41$ & $6.25 \pm 0.30$ \\
GIC & $1.38 \pm 0.21$ & $4.34 \pm 0.25$ \\
\hline
\end{tabular}

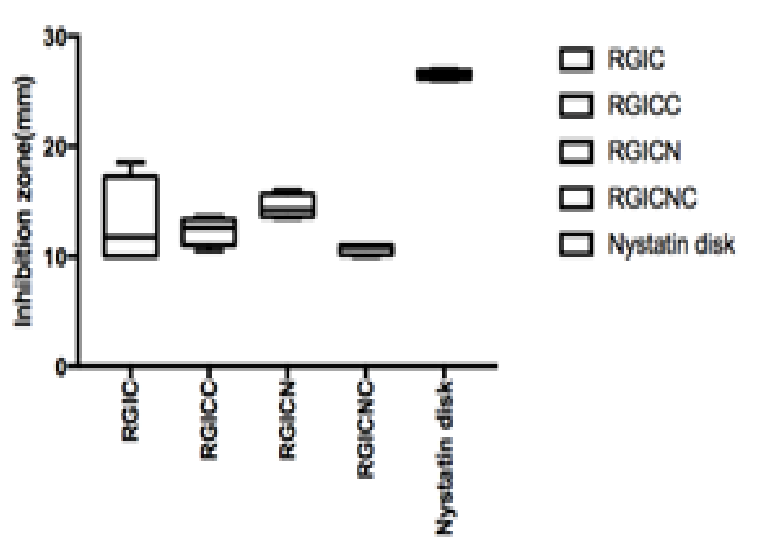

Figure 2 Inhibition zone against staphylococcus aureus (nystatin $30 \mathrm{~g}$ ).

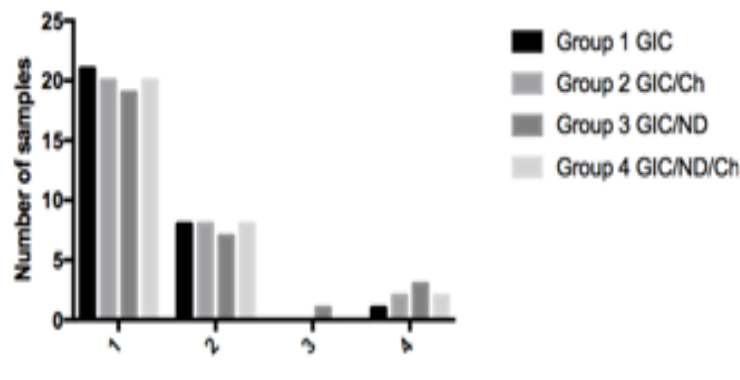

Figure 3 Comparison of microleakage observed with glass ionomer cement and chitosan or nanodiamond or chitosan/nanodiamond modified glass ionomer cement.

\section{Discussion}

\section{Dentin shear bond strength}

The results of this study suggest that optimum bond strengthening of dentin can be achieved throughout the conventional dentin conditioner/ GIC protocol, which is commonly used in dental practice. ${ }^{16}$ Although the correlation between the force and work of adhesion was noticeable for all samples. This performance can be expected because of the well-known intrinsic bio-adhesive properties of this material. Also ionic vs covalent bonding of the chitosan: therapeutic agent complex may depend on the $\mathrm{pH}$ of the environment as the $-\mathrm{COOH}$ groups in substituents of nano-diamonds ionize at alkaline $\mathrm{pH}$ and form covalent "amide" linkage at low $\mathrm{pH} .{ }^{17}$ The adequate water absorption capacity, together with its cationic nature, which promotes binding to the negative surface of skin or dentin structure, can also explain these results. This study has shown that newly developed modified materials are excellent candidates for the further development of bio-modified GIC materials. ${ }^{18}$

\section{Bio-adhesion}

Higher adhesiveness of the gels is desired to maintain an intimate contact with skin or tooth structure and results are summarized in Table 1. Bioactive modified GIC materials showed the highest adhesive force, and this result can be expected because of the well-known intrinsic bio-adhesive properties of chitosan and nano-diamonds. ${ }^{19}$

\section{Microleakage and bio-active GIC}

The results of this study reveal that incorporation of $10 \%(\mathrm{v} / \mathrm{v})$ of chitosan or nano-diamond powder or a mixture of $10 \%(\mathrm{v} / \mathrm{v})$ in glass ionomer cement did not lead to a significant increase in the micro-leakage of the materials. There was no statistically significant difference in the mean micro-leakage values between groups I II, III and IV. This could have transpired because the minor amount of chitosan or nano-diamond or a mixture of both did not hinder the formation of bond of glass ionomer with the tooth structure. These results were in harmony with the fact that Fuji IX has a coefficient of thermal expansion close to that of tooth. Only one sample showed dye penetration involving axial wall which could be due to the failure of adhesion between GC Fuji IX and tooth because of incorporation of void/ air bubble during the bulk placement of material into the cavity. Frankenberger et al..$^{20}$ reported that Fuji IX sets faster and is of higher viscosity because of finer glass particles, anhydrous polyacrylic acids of higher molecular weight and a high powder to liquid ratio. These properties may be responsible for Fuji IX exhibiting a good marginal seal. In 2007, Petri et al. ${ }^{21}$ observed the improved mechanical properties of GIC, on adding small amounts of chitosan into GIC. It was suggested that if the interfacial tension between each component is high, or in other words, the adhesion between each component is weak, the mechanical properties are poor. Therefore, an additive like chitosan would lead to formation of networks with polyacrylic acid around the inorganic particles which reduce the interfacial tension among the GIC components, thus improving mechanical performance. This effect was explained based on a model where a polymeric network binds strongly around the inorganic filler.

In 2012, Elsaka ${ }^{22}$ reported a study evaluating the antimicrobial activity and the adhesive property of dental adhesive containing various incremental concentrations of chitosan. It was reported that these properties improved upon addition of small amounts of chitosan. Upon addition of 0.12 and $0.25 \%(\mathrm{w} / \mathrm{w})$ chitosan, the micro tensile bond strength values were better compared to the control group, however there were no significant differences. Berger, et al. proposed that networks containing covalently cross linked chitosan could be 
considered as smart hydrogels undergoing a reversible discontinuous volume phase change in response to external physicochemical factors like temperature and $\mathrm{pH}^{23}$ This would in turn negate any microleakage tendency of the cement. Since in vitro studies do not reflect all variables present in the mouth, the results of the present in vitro study cannot be extrapolated to the clinical situation unless adequate clinical trials are conducted to test the in vivo efficacy of the material. Further tests should be undertaken to compare and evaluate the other strength characteristics like flexural strength, bond strength and properties like hardness, setting and working times of the experimental cement. Based on the reported benefits of improved strength and fluoride release, along with conclusions drawn from the present in vitro study of favorable microleakage results; chitosan and nanodiamond or chitosan/nanodiamond modified glass ionomer cement can be considered as a promising restorative material. It holds a place in the application of posterior occlusal restorations, in minimal invasive techniques as well as for general clinical utility in pediatric dentistry.

\section{Conclusion}

Incorporation of chitosan or nanodiamond or combination of chitosan/nano-diamond into the conventional GIC provides a promising multi-dimensional functional biomaterial with excellent properties such as increased shear bond strength, certain degree of microbiological properties, good bio-adhesion without compromising important properties such as microleakage.

\section{Acknowledgements}

None.

\section{Conflict of interest}

The author declares no conflict of interest.

\section{References}

1. Khan AS, Aamer S, Chaudhry AA, et al. Synthesis and characterizations of a fluoride-releasing dental restorative material. Mater Sci Eng C Mater Biol Appl. 2013;33(6):3458-3464.

2. Mitra SB, Oxman JD, Falsafi A, et al. Fluoride release and recharge behavior of a nano-filled resin-modified glass ionomer compared with that of other fluoride releasing materials. Am J Dent. 2011;24(6):372-378.

3. Ozak ST, Ozkan P. Nanotechnology and dentistry. Eur J Dent. 2013;7(1):145-151.

4. Chen MH. Update on dental nanocomposites. $J$ Dent Res. 2010;89(6):549-560.

5. Guggenberger R, May R, Stefan K. New trends in glass-ionomer chemistry. Biomaterials. 1998;19(6):479-483.

6. Nicholson JW. Chemistry of glass-ionomer cements: A review. Biomaterials. 1998;19(6):485-494.

7. Nicholson JW, Braybrook JH, Wasson EA. The biocompatibility of glass-poly (alkenoate) (Glass-Ionomer) cements: A review. J Biomater Sci Polym Ed. 1991;2(4):277-285.

8. Moshaverinia A, Ansari S, Movasaghi Z, et al. Modification of conventional glass-ionomer cements with vinylpyrrolidone containing polyacids, nano-hydroxy and fluoroapatite to improve mechanical properties. Dent Mater. 2008;24(10):1381-1390.
9. Khan AS, Khan M, Rehman IU. Nanoparticles, properties, and applications in glass ionomer cements in nanobiomaterials in clinical dentistry. Amstrdam, Netherlands: William Andrew Publishing; 2013.

10. Moshaverinia A, Ansari S, Moshaverinia M, et al. Effects of incorporation of hydroxyapatite and fluoroapatite nanobioceramics into conventional glass ionomer cements (GIC). Acta Biomater. 2008;4(2):432-440.

11. Uysal T, Yagci A, Uysal B, et al. Are nano-composites and nano-ionomers suitable for orthodontic bracket bonding? Eur J Orthod. 2010;32(1):78-82.

12. Basso M. Teeth restoration using a high-viscosity glass ionomer cement: The Equia® system. J Minim Interv Dent. 2011;4(3):74-76.

13. Friedl K, Hiller K, Friedl K. Clinical performance of a new glass ionomer based restoration system: A retrospective cohort study. Dent Mater. 2011;27(10):1031-1037.

14. Perchyonok V Tamara, Zhang Shengmiao, Basson Nicolaas J, et al. Evaluation of tetracycline containing chitosan hydrogels as potential dual action bio-active restorative materials capable of wound healing: in vitro approach Biointerface. Research in Applied Chemistry. 2014;4(5):843-849.

15. Perchyonok VT, Reher V, Zhang S, et al. Evaluation of nystatin containing chitosan hydrogels as potential dual action bio-active restorative materials: In Vitro approach. J Funct Biomater. 2014;5(4):259-272.

16. Perchyonok VT, Reher V, Zhang S, et al. Insights and relative effect of aspirin, naproxen and ibuprofen containing hydrogels: from design to performance as a functional dual capacity restorative material and build in free radical defence: in-vitro studies. Open Journal of Stomatology. 2014;4(2):73-83.

17. Yli Urpo H, Lassila LV, Narhi T, et al. Compressive strength and surface characterization of glass ionomer cements modified by particles of bioactive glass. Dent Mater. 2005;21(3):201-209.

18. Perchyonok VT, Zhang S, Basson NJ, et al. Insights into functional erythromycin/antioxidant containing chitosan hydrogels as potential bio-active restorative materials: structure, function and antimicrobial activity. Adv Tech Biol Med. 2014;2:116.

19. Perchyonok VT, Reher V, Zhang S, et al. Chitosan: Vitamin C containing hydrogels as a prototype functional prolonged pain management restorative material in-vitro studies. Open Jounal of Stomatology. 2014;4(8):389-401.

20. Frankenberger R, Sindel J, Kramer N. Viscous glass-ionomercements: a new alternative to amalgam in the primary dentition? Quintessence Int. 1997;28(10):667-676.

21. Petri DFS, Donega J, Benassi AM, et al. Preliminary study on chitosan modified glass ionomer restoratives. Dent Mater. 2007;23(8):1004 1010 .

22. Elsaka ES. Antibacterial activity and adhesive properties of a chitosan-containing dental adhesive. Quintessence Int. 2012;47(7):603-613.

23. Kean T, Thanou M. Biodegradation, biodistribution and toxicity of chitosan. Adv Drug Deliv Rev. 2010;62(1):3-11. 